\title{
Steady-State Postural Response to Neuromuscular and Proprioceptive Training Program in Active Middle-Aged Women
}

\author{
Mohammadreza Rezaeipour ${ }^{1,{ }^{*}}$ and Gennady Leonidovich Apanasenko ${ }^{2}$ \\ ${ }^{1}$ Sports Sciences Department, University of Sistan and Baluchestan, Zahedan, Iran \\ ${ }^{2}$ Ukrainian Center of Sports Medicine, NMAPE, Kiev, Ukraine \\ "Corresponding author: Assistant Professor, Sports Sciences Department, University of Sistan and Baluchestan, Postal Box: 98135-987, Postal Code: 9816745639, Zahedan, Iran. \\ Tel. +98-9153414047, Email: rezaeipour@ped.usb.ac.ir
}

Received 2019 May 16; Revised 2019 September 20; Accepted 2019 October 26.

\begin{abstract}
Background: Exercise training appears to have an essential role in maintaining motor responses.

Objectives: This study aimed to explore the steady-state postural response to neuromuscular and proprioceptive training (NPT) in active middle-aged women.

Methods: Forty-four active women (mean age, $53.2 \pm 6.1$ years) completed this cross-sectional study. During the 12-week study, the NPT group $(n=20)$ performed the NPT program, while the control group $(n=24)$ followed the usual warm-up routines. Postural stability in different visual conditions was measured in the anterior-posterior (AP) and medial-lateral (ML) planes during quiet standing on a foam mat at baseline and after the intervention using a force platform.

Results: The NPT could significantly improve postural balance in the ML plane with Eyes Open $(\mathrm{EO})(\mathrm{P}=0.005)$ and Eyes Closed (EC) $(\mathrm{P}=0.004)$ and in the AP plane with $\mathrm{EO}(\mathrm{P}=0.006)$ and $\mathrm{EC}(\mathrm{P}=0.003)$. Estimates of mean CoP total velocity with $\mathrm{EO}(\mathrm{P}=0.005)$ and $\mathrm{EC}(\mathrm{P}=0.004)$ also revealed significant interaction effects on any CoP parameter. However, values of partial eta squared effect size only in the AP direction indicated a large, powerful significance for the EC condition.

Conclusions: The NPT is effective in improving postural steadiness in middle-aged women. Therefore, it might have a direct effect on decreasing the falling risk of active middle-aged women.
\end{abstract}

Keywords: Anthropometry, Postural Balance, Exercise, Posture, Women’s Health

\section{Background}

Postural steadiness is the ability to stand upright using the coordination and integration of data provided by the sensorimotor system (1). Researchers have shown that postural control deficiencies in the aging process can be linked to several factors, including deterioration of sensory systems (2), proprioception of lower extremities (3), and reduced muscle strength of lower extremities (4). With the beginning of the fourth decade of life, instability in the postural balance system gradually begins (5) and it gets worse as the age rises $(2,5)$, making it an essential social problem (6). While not all falls cause injuries, about $20 \%$ necessitate medical care, $5 \%$ lead to fractures, and 5\% $10 \%$ cause other serious injuries, such as severe head injury, dislocation, joint sprain, and soft tissue damage (7). After cardiovascular diseases, cancer, stroke, and pulmonary disorders, accidental damage is the fifth leading cause of death in the elderly and two-thirds of these deaths are due to falls (8). Therefore, to begin administering simple and low-cost treatment, early treatment of risk factors is essential.

The combination of various exercises such as balance, dynamic, plyometric training, strengthening, running, agility, and stretching, irrespective of specific exercises, is a source of neuromuscular and proprioceptive training (NPT) (9). The NPT integration in the warm-up is commonly utilized by some young athletes and has a positive effect on postural stability (10-12). However, it has not yet been proven whether the NPT integration in routine training sessions improves the ability to maintain balance in people of middle-age. The mean velocity of movement, which is a parameter of the center of pressure (CoP), can be differentiated correctly in various experimental situations and it also has a minimum standardized coefficient of interpersonal variation, i.e., the lowest reproducibility error (13). Therefore, this study used the measurements of mean CoP velocity in various directions to assess the postural stability of active middle-aged women. It was hypothesized that significant improvements in postural stability could be ob- 
tained through a 12-week NPT program for middle-aged women.

\section{Objectives}

The objective of this study was to assess the impact of the NPT program, rather than general warm-up routines, on postural steadiness during quiet standing in active middle-aged women.

\section{Methods}

\subsection{Participants}

This cross-sectional study was conducted in autumn 2018 on 54 women selected through questionnaires and interviews at the Sports Medicine Center (Kyiv, Ukraine). The participants were divided into two groups by random distribution (using a manual lottery). Ten women were omitted throughout the study, seven of whom belonged to the NPT group. Therefore, $81 \%$ of participants (44 people) completed the study. The final sample size was 20 people in the NPT group and 24 people in the control group and we utilized their information in this study. Attending at least three exercise sessions per week and ages of 45 to 65 years were considered as the inclusion criteria. Neurological disease, cardiovascular disease, diabetes, Parkinson's disease, musculoskeletal system disease (such as pain in the lower back and lower extremity), taking medication, and a history of falls were the exclusion criteria according to the physician's diagnosis (14-16)

\subsection{Intervention}

Subjects in the NPT group had a 20-min warm-up period with the NPT program and the control group performed body warm-up routines such as strengthening, stretching, and dynamic exercises for $20 \mathrm{~min}$ (Figure 1.). Each training session included a 45-min weight training, based on the recommendations of the American Heart Association and the American College of Sports Medicine (17, 18), and finally ended with a 10-min cool-down (Figure 1). The training sessions were the same in both groups and were conducted three times a week for 12 weeks. The NPT program was organized and monitored by the research team as provided in Table 1 . Before the test, instructors and athletes became acquainted with the NPT and each training session was taught.

\begin{tabular}{|c|c|c|}
\hline Exercise & Distance & Repetitions/Time \\
\hline \multicolumn{3}{|l|}{ Warm-up } \\
\hline Shuttle run & $45 \mathrm{~m}$ & 1 \\
\hline Backward running & $45 \mathrm{~m}$ & 1 \\
\hline \multicolumn{3}{|l|}{ Stretching } \\
\hline Calf stretch & - & $2 \times 30 s$ \\
\hline Quadriceps stretch & - & $2 \times 30 s$ \\
\hline Hamstring stretch & - & $2 \times 30 s$ \\
\hline Inner thigh stretch & - & $2 \times 30 s$ \\
\hline Hip flexor stretch & - & $2 \times 30 s$ \\
\hline \multicolumn{3}{|l|}{ Strengthening } \\
\hline Walking lunges & $18 \mathrm{~m}$ & 2 passes \\
\hline Single-toe raises & - & 30 , bilaterally \\
\hline \multicolumn{3}{|l|}{ Plyometrics } \\
\hline Lateral hops & 2-to 6-in cone & $30 \mathrm{~s}$ \\
\hline Forward hops & 2-to 6-in cone & $30 \mathrm{~s}$ \\
\hline Single-legged hops & 2- to 6-in cone & $30 \mathrm{~s}$ \\
\hline Vertical jumps & - & $30 \mathrm{~s}$ \\
\hline Scissors jumps & - & $30 \mathrm{~s}$ \\
\hline \multicolumn{3}{|l|}{ Agilities } \\
\hline Diagonal runs & $36 \mathrm{~m}$ & 1 \\
\hline Bounding runs & $41 \mathrm{~m}$ & 1 \\
\hline
\end{tabular}

Abbreviations: m, meter; s, second

\subsection{Method Management}

The participants were asked to preserve typical food consumption, two liters of water consumption per day, and eight hours of sleep. They were all informed of the study and provided their written informed consent prior to data collection after presenting a medical certificate proving that the intervention was not contraindicated. The Institutional Ethics Committee [NMAPE, (2018) No. 04112-12] approved the forms of informed consent and the use of human ethics based on the principles of the Helsinki Declaration. The absence of each participant was monitored weekly.

\subsection{Measurements}

Table 2 shows the baseline descriptive characteristics of both groups. After anthropometric evaluations, postural stability was conducted among participants by measuring the mean CoP velocity during quiet standing (barefoot) before and after the 12-week intervention. A force plate (Kistler 9286AA, Kistler Group, Switzerland) was used to study postural steadiness during quiet standing on a 
20 minutes warming-up in NPT group consisted of two basic warm-up activities, five stretching techniques for the trunk and lower extremity, two strengthening exercises, five plyometric activities, and two soccer-specific agility drills (Table 1 ).
20 minutes warming-up in control group consisted of 10 reps each neck tilts, neck and rotations, chest expansions, side arm raises, arm and hip rotations, hops on the spot and side-to-side (routine warm-up).

Figure 1. The general aspects of the two exercises programs (NPT vs. control).

foam mat with eyes open (EO) and eyes closed (EC) (5). This method was formerly applied to display the impacts of exercise on postural steadiness $(19,20)$, and was expected to be sensitive to alterations in postural steadiness (21). The length of each record in any visual condition was 30 seconds, followed by a short rest period for two minutes. All measurements were performed twice for each visual state, at a sampling rate of $200 \mathrm{~Hz}$ in the same order by the same trained instructors in the anterior-posterior (AP) and medial-lateral (ML) planes. During the test, participants were instructed to stand in a quiet position as their home or workplace and look directly ahead at a black spot $(2 \times 2$ $\mathrm{cm})$ at $2 \mathrm{~m}$ on the wall when conducting EO tests.

\begin{tabular}{|c|c|c|c|}
\hline Variable & Control Group $(\mathrm{N}=\mathbf{2 4})$ & NPT Group $(\mathrm{N}=20)$ & P Value \\
\hline Age, $y$ & $52.00 \pm 5.9$ & $54.4 \pm 6.4$ & 0.28 \\
\hline Weight, kg & $64.6 \pm 9.4$ & $63.2 \pm 10.8$ & 0.41 \\
\hline Height, cm & $1.60 \pm 0.2$ & $1.58 \pm 0.2$ & 0.54 \\
\hline BMI, $\mathrm{kg} / \mathrm{m}^{2}$ & $25.2 \pm 4.5$ & $25.4 \pm 2.7$ & 0.51 \\
\hline
\end{tabular}

\subsection{Statistical Analysis}

The IBM SPSS Ver. 21.0 for Windows was used for statistical analysis. All data were presented as means \pm standard deviations. The MATLAB application (Stable release: R2019a; Mathworks, USA) was used to filter data by Butterworth Filter (a fourth-order low pass filter) with a cutoff frequency of $7 \mathrm{~Hz}(22,23)$. The normality of data distribution was confirmed with the Kolmogorov-Smirnov test. To compare the impact of interventions at any eye state, we used a two-way mixed factorial ANOVA [group $\times$ time; betweensubject = group (NPT and Control groups); within-subject = time (before and after the study)]. Moreover, a paired $t$-test with Bonferroni correction was performed to compare the pre and post-study values for each measurement of CoP swing. Bonferroni adjustments were calculated by dividing 0.05 by the number of analyses conducted. The statistical significance level was adjusted to $\leq 0.008$ using the Bonferroni method (Bonferroni adjustment $=0.05 / 6$ $=0.0083)$. Partial eta squared $\left(\eta_{\mathrm{p}}{ }^{2}\right)$ effect sizes (24) were small, medium, and large based on the calculated values of $0.01,0.06$, and 0.14 , respectively (25). Statistical significance was considered at $\mathrm{P}<0.05$ with a confidence interval of $95 \%$.

\section{Results}

As shown in Table 2, at the beginning of the study, there was no significant difference in the examined variables between the groups $(\mathrm{P}>0.05)$.

Of 10 participants who left the study, two were due to health reasons, three due to loss of interest, four due to lack of time, and one due to unknown reasons. Table 3 shows postural data in both planes with EO and EC.

Prior to the study, there were no significant differences between the study groups under $\mathrm{EO}$ in terms of mean CoP total velocity $(\mathrm{V})(\mathrm{P}=0.13)$, mean CoPvelocity in direction of $\operatorname{ML}(\mathrm{P}=0.12)$ and direction of $\mathrm{AP}(\mathrm{P}=0.14)$. In addition, the comparison of $\mathrm{V}(\mathrm{P}=0.11)$, mean CoP velocity in direction of $\mathrm{ML}(\mathrm{P}=0.12)$ and direction of $\mathrm{AP}(\mathrm{P}=0.11)$ did not show significant differences during EC (Table 3 ).

After the 12-week NPT program, postural stability during quiet standing improved in the ML plane with EO (P 


\begin{tabular}{|c|c|c|c|c|c|c|}
\hline \multirow{2}{*}{$\begin{array}{l}\text { Center of Pressure } \\
\text { Velocity }\end{array}$} & \multirow{2}{*}{ Condition } & \multirow{2}{*}{ Group } & \multirow{2}{*}{ Pretest } & \multirow{2}{*}{ Posttest } & \multicolumn{2}{|c|}{ Group-Time Interaction } \\
\hline & & & & & PValue & $\eta_{\mathbf{p}}{ }^{2}$ ES \\
\hline \multirow{4}{*}{$\begin{array}{l}\text { ML direction } \\
\left(\mathrm{cm} . \mathrm{s}^{-1}\right)\end{array}$} & \multirow{2}{*}{$\mathrm{EO}$} & NPT & $4.31(0.90)$ & $4.16(0.45)$ & \multirow{2}{*}{$0.005^{*}$} & \multirow{2}{*}{0.01} \\
\hline & & Control & $4.34(045)$ & $4.30(0.55)$ & & \\
\hline & \multirow{2}{*}{$\mathrm{EC}$} & NPT & $5.25(0.72)$ & $5.06(0.39)$ & \multirow{2}{*}{$0.003^{*}$} & \multirow{2}{*}{0.06} \\
\hline & & Control & $5.27(0.59)$ & $5.21(0.64)$ & & \\
\hline \multirow{4}{*}{$\begin{array}{l}\text { AP direction } \\
\left(\mathrm{cm} \cdot \mathrm{s}^{-1}\right)\end{array}$} & \multirow{2}{*}{$\mathrm{EO}$} & NPT & $8.82(0.85)$ & $8.53(0.34)$ & \multirow{2}{*}{$<0.001^{*}$} & \multirow{2}{*}{0.09} \\
\hline & & Control & $8.86(0.38)$ & $8.82(0.68)$ & & \\
\hline & \multirow{2}{*}{$\mathrm{EC}$} & NPT & $12.47(0.70)$ & $12.15(0.34)$ & \multirow{2}{*}{$<0.001^{*}$} & \multirow{2}{*}{0.14} \\
\hline & & Control & $12.50(0.54)$ & $12.45(0.58)$ & & \\
\hline \multirow{3}{*}{ Total $\left(\mathrm{cm} . \mathrm{s}^{-1}\right)$} & EO & Control & $11.20(0.53)$ & $11.15(0.61)$ & $<0.001^{*}$ & 0.04 \\
\hline & \multirow{2}{*}{ EC } & NPT & $15.14(1.00)$ & $14.87(0.52)$ & \multirow{2}{*}{$0.004^{*}$} & \multirow{2}{*}{0.08} \\
\hline & & Control & $15.20(0.79)$ & $15.14(0.66)$ & & \\
\hline
\end{tabular}

Abbreviations: AP, mean CoP velocity in the anterior-posterior direction $\left(\mathrm{cm} . \mathrm{s}^{-1}\right)$; EC, eyes closed condition; EO, eyes open condition; ML, mean CoP velocity in the mediolateral direction $\left(\mathrm{cm}^{-1} \mathrm{~s}^{-1}\right)$ NPT, neuromuscular and proprioceptive training; $\eta_{\mathrm{p}}{ }^{2} \mathrm{ES}$, effect sizes by partial eta squared

$=0.005)$ and $\mathrm{EC}(\mathrm{P}=0.004)$ and the AP plane with EO $(\mathrm{P}$ $=0.006)$ and $\mathrm{EC}(\mathrm{P}=0.003)$. The assessment of mean CoP total velocity with EO $(\mathrm{P}=0.005)$ and $\mathrm{EC}(\mathrm{P}=0.004)$ also showed a significant interaction effect on any parameter of the CoP. The measures of CoP velocity depending on statistical analysis showed the significant impact of group-time interaction on the mean CoP velocity in the plane of ML for EO and EC (small to medium effect size). a significant interaction effect was seen in the AP plane for EO and EC and the $V$ parameter for EO and EC, while, their effect size in the AP plane (medium to large effect size) and the $V$ parameter (small to medium effect size) was different. (Table 3 ).

\section{Discussion}

It is important to note that an irregular exercise program will not improve traditional markers of physiological performance and fitness in the elderly (26). Therefore, a regular exercise program was considered in this study. The close link between obesity and postural instability has been shown in several studies. However, only have a few studies been conducted on non-obese and postural control among middle-aged women (23). Regardless of the effect of BMI as one of the body composition parameters on postural balance, the objective of this study was to assess the impact of a 12-week NPT program, rather than general warm-up routines, on postural steadiness during quiet standing in active middle-aged women. The differences in participants' BMIs, as shown in Table 2 (BMIs of 28.1 and 22.7 in the NPT group with a mean of $25.4 \pm 2.7$ ), seem to confirm the effects of the NPT and its application on all middle-aged women. It is widely recognized that postural instability is one of the characteristics of aging $(27,28)$. The reductions observed in this study were incompatible with age-related alterations in CoP. Given the reliability (29) and sensitivity (19) of the mean CoP velocity, the results of the current study confirmed the hypothesis and the positive effect of NPT on postural steadiness.

The NPT enables successful interactions between the components of the motor control system to maintain the human body in the upright stance (11). Postural control enhancements caused by the NPT are often discussed in the context of adapting to the motor control system (30). However, many controlled studies have shown contradictory results on sports-induced changes in the motor control system in healthy individuals $(31,32)$. This study showed the age-related deterioration of postural instability was reversed by the NPT and the program improved balance ability and postural control in active middle-aged women.

Some researchers believe that one of the factors contributing to the improvement of postural stability during exercise is the rising body temperature (33). Therefore, the positive effects of exercise cannot continue for a long time. These findings contradict the results of the present study. This study also showed that visual perception as an ambient system can influence the postural steadiness of middle-aged women and confirmed the findings of other researchers who suggested that visual conditions could affect stability and balance (34).

Since we studied merely women, the NPT effects on 
men are unclear. Therefore, research on men is proposed. Other limitations of this study were that the consequences of NPT cessation after the study remained unknown and the mean CoPvelocity was measured by one force platform during an upright stance. As pointed out earlier, BMI characteristics were not evaluated and this could be considered another limitation.

\subsection{Conclusions}

The NPT is linked to significant improvements in static situation and postural stability. A 12-week NPT could reduce mean CoP velocity during the static condition of lateral and anterior-posterior stability in older adults. The study of postural stability caused by aging during quiet stance can lead to improving health, assessment, or rehabilitation of impaired balance to increase the quality of life of older adults.

\section{Footnotes}

Authors' Contribution: Mohammadreza Rezaeipour and Gennady Leonidovich Apanasenko made substantial contributions to the conception, design, and acquisition, analysis, and interpretation of data. Mohammadreza Rezaeipour was involved in the drafting of the manuscript and revising it critically for important intellectual content. All the authors read and approved the final manuscript.

Conflict of Interests: None.

Ethical Approval: Participants were informed of the study. After receiving a medical certificate declaring that the intervention did not have contraindications, the participants provided their written informed consent prior to data collection. The Institutional Ethics Committee [NMAPE, (2018) No. 04112-12] approved the forms of informed consent and the use of human ethics based on the principles of the Helsinki Declaration.

\section{Funding/Support: None.}

\section{References}

1. Forssberg H, Nashner LM. Ontogenetic development of postural control in man: Adaptation to altered support and visual conditions during stance. J Neurosci. 1982;2(5):545-52. [PubMed: 7077364]. [PubMed Central: PMC6564264].

2. Abrahamova D, Hlavacka F. Age-related changes of human balance during quiet stance. Physiol Res. 2008;57(6):957-64. [PubMed: 18052683].

3. Skinner HB, Barrack RL, Cook SD. Age-related decline in proprioception. Clin Orthopaedics Related Res. 1984;1984(184):208-11. doi: 10.1097/00003086-198404000-00035.

4. Menz HB, Morris ME, Lord SR. Foot and ankle characteristics associated with impaired balance and functional ability in older people. J Gerontol A Biol Sci Med Sci. 2005;60(12):1546-52. doi: 10.1093/gerona/60.12.1546. [PubMed: 16424286].
5. Sieńko-Awierianów E, Lubkowska A, Kolano P, Chudecka M. Postural stability and risk of falls per decade of adult life - a pilot study. Anthropol Rev. 2018;81(1):102-9. doi: 10.2478/anre-2018-0008.

6. Błaszczyk JW, Czerwosz L. Stabilność posturalna w procesie starzenia. Gerontologia Polska. 2005;13(1):25-36.

7. Kannus P, Sievanen H, Palvanen M, Jarvinen T, Parkkari J. Prevention of falls and consequent injuries in elderly people. Lancet. 2005;366(9500):1885-93. doi: 10.1016/S0140-6736(05)67604-0. [PubMed: 16310556].

8. Rubenstein LZ. Falls in older people: Epidemiology, risk factors and strategies for prevention. Age Ageing. 2006;35 Suppl 2:ii37-41. doi: 10.1093/ageing/afl084. [PubMed:16926202].

9. Mandelbaum BR, Silvers HJ, Watanabe DS, Knarr JF, Thomas SD, Griffin LY, et al. Effectiveness of a neuromuscular and proprioceptive training program in preventing anterior cruciate ligament injuries in female athletes: 2-year follow-up. Am J Sports Med. 2005;33(7):1003-10. doi: 10.1177/0363546504272261. [PubMed: 15888716].

10. Bird SP, Stuart W. Integrating balance and postural stability exercises into the functional warm-up for youth athletes. Strength Cond J. 2012;34(3):73-9. doi: 10.1519/SSC.0b013e31824f175e.

11. Ondra L, Natesta P, Bizovska L, Kubonova E, Svoboda Z. Effect of inseason neuromuscular and proprioceptive training on postural stability in male youth basketball players. Acta Gymnica. 2017;47(3):144-9. doi: 10.5507/ag.2017.019.

12. Pasanen K, Parkkari J, Pasanen M, Kannus P. Effect of a neuromuscular warm-up programme on muscle power, balance, speed and agility: A randomised controlled study. Br J Sports Med. 2009;43(13):1073-8. doi: 10.1136/bjsm.2009.061747. [PubMed: 19622526].

13. Raymakers JA, Samson MM, Verhaar HJ. The assessment of body sway and the choice of the stability parameter(s). Gait Posture. 2005;21(1):48-58. doi: 10.1016/j.gaitpost.2003.11.006. [PubMed: 15536033].

14. Sun W, Zhang C, Song Q, Li W, Cong Y, Chang S, et al. Effect of 1year regular Tai Chi on neuromuscular reaction in elderly women: A randomized controlled study. Res Sports Med. 2016;24(2):145-56. doi: 10.1080/15438627.2015.1126280. [PubMed: 27161956].

15. Safi K. Human postural stability analysis: Application to Parkinsonian subjects [Dissertation]. University of Paris-Est; 2016.

16. Rezaeipour M, Apanasenko GL. Effects of overweight and obesity on postural stability of aging females. Middle East J Rehabil Health. 2018;5(4). doi: 10.5812/mejrh.81617.

17. Alves AJ, Viana JL, Cavalcante SL, Oliveira NL, Duarte JA, Mota J, et al. Physical activity in primary and secondary prevention of cardiovascular disease: Overview updated. World J Cardiol. 2016;8(10):57583. doi: 10.4330/wjc.v8.i10.575. [PubMed: 27847558]. [PubMed Central: PMC5088363].

18. Haskell WL, Lee IM, Pate RR, Powell KE, Blair SN, Franklin BA, et al. Physical activity and public health: updated recommendation for adults from the American College of Sports Medicine and the American Heart Association. Circulation. 2007;116(9):1081-93. doi: 10.1161/CIRCULATIONAHA.107.185649. [PubMed: 17671237].

19. Mello RG, Oliveira LF, Nadal J. Anticipation mechanism in body sway control and effect of muscle fatigue. I Electromyogr Kinesiol. 2007;17(6):739-46. doi: 10.1016/j.jelekin.2006.08.011. [PubMed: 17142060].

20. Vuillerme N, Forestier N, Nougier V. Attentional demands and postural sway: The effect of the calf muscles fatigue. Med Sci Sports Exerc. 2002;34(12):1907-12. doi: 10.1097/00005768-200212000-00008. [PubMed: 12471295].

21. Era P, Sainio P, Koskinen S, Haavisto P, Vaara M, Aromaa A. Postural balance in a random sample of 7,979 subjects aged 30 years and over. Gerontology. 2006;52(4):204-13. doi: 10.1159/000093652. [PubMed: 16849863].

22. Rezaeipour M. Evaluation of postural stability in overweight and obese middle-aged men. Turk J Med Sci. 2018;48(5):1053-7. doi: 10.3906/sag-1709-108. [PubMed: 30384575]. 
23. Kovacikova Z, Svoboda Z, Neumannova K, Bizovska L, Cuberek $\mathrm{R}$, Janura M. Assessment of postural stability in overweight and obese middle-aged women. Acta Gymnica. 2014;44(3):149-53. doi: 10.5507/ag.2014.015.

24. Gabizon H, Press Y, Volkov I, Melzer I. The effects of pilates training on balance control and self-reported health status in communitydwelling older adults: A randomized controlled trial. J Aging Phys Act. 2016;24(3):376-83. doi: 10.1123/japa.2014-0298. [PubMed: 26540737].

25. Richardson JTE. Eta squared and partial eta squared as measures of effect size in educational research. Educ Res Rev. 2011;6(2):135-47. doi: 10.1016/j.edurev.2010.12.001.

26. Chodzko-Zajko WJ, Proctor DN, Fiatarone Singh MA, Minson CT, Nigg CR; American College of Sports Medicine, et al. Exercise and physical activity for older adults. Med Sci Sports Exerc. 2009;41(7):1510-30. doi: 10.1249/MSS.0b013e3181a0c95c. [PubMed: 19516148].

27. Ghahramani M, Naghdy F, Stirling D, Naghdy G, Potter J. Impact of age on body postural sway. TENCON 2015-2015 IEEE Region 10 Conference. 2015. p. 1-6.

28. Teasdale N, Simoneau M. Attentional demands for postural control: The effects of aging and sensory reintegration. Gait Posture. 2001;14(3):203-10. doi: 10.1016/s0966-6362(01)00134-5. [PubMed: $11600323]$.
29. Lin D, Seol H, Nussbaum MA, Madigan ML. Reliability of COPbased postural sway measures and age-related differences. Gait Posture. 2008;28(2):337-42. doi: 10.1016/j.gaitpost.2008.01.005. [PubMed 18316191].

30. Hewett TE, Paterno MV, Myer GD. Strategies for enhancing proprioception and neuromuscular control of the knee. Clin Orthop Relat Res. 2002;(402):76-94. doi: 10.1097/00003086-200209000-00008. [PubMed: 12218474].

31. Zech A, Hubscher M, Vogt L, Banzer W, Hansel F, Pfeifer K. Balance training for neuromuscular control and performance enhancement: A systematic review.JAthl Train. 2010;45(4):392-403. doi: 10.4085/10626050-45.4.392. [PubMed: 20617915]. [PubMed Central: PMC2902034].

32. Crilly RG, Willems DA, Trenholm KJ, Hayes KC, DelaquerriereRichardson LF. Effect of exercise on postural sway in the elderly. Gerontology. 1989;35(2-3):137-43. doi: 10.1159/000213012. [PubMed: 2792781].

33. Mense S. Effects of temperature on the discharges of muscle spindles and tendon organs. Pflugers Arch. 1978;374(2):159-66. doi 10.1007/bfo0581297. [PubMed: 566425].

34. Kejonen PK. Body movements during postural stabilization: Measurements with a motion analysis system [dissertation]. Finland: University of Oulu; 2004 